Europhys. Lett., 2 (2), pp. 83-85 (1986)

\title{
Connections between Deviations from Lorentz Transformation and Relativistic Energy-Momentum Relation.
}

\author{
K. SvOzIL \\ Institute for Theoretical Physics, Technical University Vienna \\ Karlsplatz 13/136, A-1040 Vienna, Austria \\ (received 10 February 1986; accepted 30 April 1986) \\ PACS. 03.30. - Special relativity. \\ PACS. 03.65. - Quantum theory; quantum mechanics.
}

\begin{abstract}
Violations of Lorentz transformation for space-time scales render corrections to relativistic energy-momentum relations and vice versa.
\end{abstract}

Some recent articles [1-3] on tests of the special theory of relativity (STR) were motivation to write down a result obtained several years ago: that the transformation properties of intrinsic space and time scales depend on the dispersion relation (that is the energy-momentum relation) of a particular system; and vice versa: a deviation from the Lorentz transformation would result in nonrelativistic transformation of energy and mass, as well as nonrelativistic energy-momentum relations.

Throughout this communication, Einstein's original operationalizations for the concepts [4] of simultaneity and two-way velocity of light are applied. This enables a better comparison between STR and alternate theories. A quantized two-state system $\{|1\rangle,|2\rangle\}$ is considered, which can serve as a clock (present-day state of the art atomic clocks can be described very similarly). The system starts out in a state $|1\rangle$, which is no eigenstate of the Hamiltonian $H$. It will, therefore, undergo oscillations between $|1\rangle$ and $|2\rangle$. Assume an arbitrary real number $t_{A}$ for the initial time, and an arbitrary real number $t_{B}>t_{A}$ for a later time. Denoting the amplitudes of the states by $c_{1}(t)=\langle 1 \mid \psi(t)\rangle$ and $c_{2}(t)=\langle 2 \mid \psi(t)\rangle$; and assuming symmetric transition rules $H_{11}=H_{22}$ and $H_{12}=H_{21}$, the Schrödinger equation reads

$$
\begin{aligned}
& i \frac{\mathrm{d}}{\mathrm{d} t} c_{1}=H_{11} c_{1}+H_{12} c_{2}, \\
& i \frac{\mathrm{d}}{\mathrm{d} t} c_{2}=H_{21} c_{1}+H_{22} c_{2} .
\end{aligned}
$$

Its solutions are oscillations in Hilbert space. With the above initial condition $\left|c_{1}\left(t_{A}\right)\right|^{2}=1-\left|c_{2}\left(t_{B}\right)\right|^{2}=1$, a short calculation yields

$$
\left|c_{1}\left(t_{B}\right)\right|^{2}=\cos ^{2}\left[H_{12}\left(t_{A}-t_{B}\right)\right] .
$$


The time span $t_{B}-t_{A}$ has been defined arbitrarily; and (2) can be measured. Hence, the energy scale of $H$ has to be calibrated, such that (2) is satisfied. This condition sets the energy scale in a particular frame of reference.

Now consider two reference frames, $a$ and $b$, both carrying (quantized) clocks with them. Suppose an observer in $a$ watches the clock in $b$, as $b$ passes with a velocity $v$. The observer is comparing the times of synchronized clocks resting in $a$ with the time of the clock resting in $b$. Since the quantum states of the clocks can be described similarly in both systems, state vectors and amplitudes can be identified: for instance $\left|1_{\alpha}\right\rangle=\left|1_{b}\right\rangle=|1\rangle$ and $c_{1}\left(t_{A \alpha}\right)=c_{1}\left(t_{A b}\right)$. Since in both frames a Schrödinger equation similar to (1) can be written down, the dynamics is the same, and we obtain by identifying the amplitudes for state $|1\rangle$,

$$
\cos ^{2}\left[H_{12 a}\left(t_{A \alpha}-t_{B a}\right)\right]=\cos ^{2}\left[H_{12 b}\left(t_{A b}-t_{B b}\right)\right]
$$

The indices $a$ and $b$ mean «measured in $a$ and $b »$. For infinitesimal time difference $\Delta t=t_{B}-t_{A} \rightarrow \mathrm{d} t$, eq. (3) yields

$$
\frac{\mathrm{d} t_{a}}{\mathrm{~d} t_{b}}=\frac{H_{12 b}}{H_{12 a}}=: \gamma
$$

Next, some velocity $c$ (prefereably a sound velocity or the maximum signal velocity) is defined to be constant in all reference frames, in particular $c_{a}=c_{b}=c$. With eq. (4), this covention immediately yields the dilatation laws for space scales parallel to $v$ :

$$
\frac{\mathrm{d} x^{\alpha}}{\mathrm{d} x^{b}}=\gamma .
$$

Now, the question is, what is the $\gamma$-factor in (4) and (5), defined by the ratio between the nondiagonal elements of the Hamiltonians in the two frames $a$ and $b$ ?

An immediate thought (yielding Galileian-type transformation properties) would be to identify $H_{12 a}$ with $H_{12 b}$ by definition, yielding $\gamma=1$. As has been shown [5], this would result in noncovariant transformation laws for $H$, although the phenomenology may be the same as for STR.

When, for some reasons, it is desireable to obtain symmetric transformation laws (which need not necessarily be Lorentz covariant), the usual energy-scale calibration may be applied: Assume an object (for instance the clock described above) at rest in a reference frame $a$, with an associate energy $E_{\alpha}$. When it is transferred to another reference frame $b$ such that it is at rest there, then the energy scale in $a$ and $b$ have to be calibrated ( synchronized»), such that $E_{a}=E_{b}$ for this object. This principle is identical to saying that particle rest masses are the same for all reference frames. Such an energy calibration convention is «evident», although not unique. It completes Einstein's synchronization conventions for space and time scales.

Going back to the clock model described above, the clock in $b$ moves with a velocity $v$ and an associated momentum $p$ with respect to $a$. By identifying $H_{12}=E(p)$, one obtains

$$
r=\frac{\mathrm{d} t_{a}}{\mathrm{~d} t_{b}}=\frac{\mathrm{d} x_{a}}{\mathrm{~d} x_{b}}=\frac{E(0)}{E(p)} .
$$

That these dilatation laws do not necessarily reproduce Lorentz-type transformation properties is straightforward. In fact, only for a particular type of dispersion relation, where $E(p)=\left[E_{0}^{2}+c^{2} p^{2}\right]^{\frac{1}{2}}$, is Lorentz covariance reproduced. For a «cellular ether», composed out of cells [6], coupled linearly to their equilibrium position and their immediate neighbors (locally), this relation is satisfied for weak analytic forces. For media with different type of 
dispersion relations (such as soliton phenomena), intrinsic space-time scales transform differently from STR. On the other hand, deviations from the Lorentz transformation would render nonrelativistic dispersion relations. For example, from muon lifetime experiments $[3,7]$, a bound on the following dispersion relation ansatz can be given: when $E(p)=\left[E_{0}^{2}+c^{2} p^{2}+\alpha\left(c^{2} p^{2} / E_{0}\right)^{2}\right]^{\frac{1}{2}}$, then

$$
x \leqslant 10^{-3} \text {. }
$$

In conclusion it can be said that different dispersion relations (energy-momentum relations) yield different transformation properties for intrinsic space-time co-ordinates. The same holds true vice versa, that is space-time transformation laws allow the specification of energy-momentum relations

The author gratefully acknowledges discussions with some members of the LBLBerkeley staff in 1982/83, and the University of Vienna relativity group. This work has been supported by the Austrian Ministry for Science and Research, project number 19.153/3$26 / 85$.

\section{REFERENCES}

[1] A. K. A. Maciel and J. Tiomno: Phys. Rev. lett., 55, 143 (1985),

[2] E. Fischbach et al.: Phys. Rev. D, 32, 154 (1985).

[3] D. W. MacArthur: Phys. Rev. A, 33, 1 (1986).

[4] A. Einstein: Ann. Phys., 17, 891 (1905).

[5] R. Mansolri and R. U. SEXL: Gen. Relativ. Gravit., 8, 497 (1977).

[6] D. Richardson: J. Comp. Sys. Sci, , 6, 373 (1972).

[7] J. BAILERY et al.: Nature (London), 268, 301 (1977). 